\title{
MICROANÁLISE DE INCLUSÕES NÃO METÁLICAS DE ARTEFATOS FERROSOS DA FÁBRICA DE FERRO DE IPANEMA VISANDO ESTABELECER A ASSINATURA QUÍMICA DO SEU PROCESSO PRODUTIVO*
}

\author{
Elmer Antonio Mamani Calcina ${ }^{1}$ \\ Fernando José Gomes Landgraf ${ }^{2}$ \\ Cesar Roberto de Farias Azevedo 3
}

\section{Resumo}

Análise de conglomerados e caracterização microestrutural, incluindo microanálise química, foram usadas para investigar as inclusões não-metálicas de amostras ferrosas da Real Fabrica de Ferro de Ipanema e da Ponte D. Pedro II (localizada na Bahia e produzida na Escócia); e de artefatos ferrosos de sítios arqueológicos de São Miguel das Missões (Rio Grande do Sul) e de Afonso Sardinha (Sorocaba, coleção da Prof. ${ }^{a}$ Margarida Andreatta) com o objetivo de estabelecer a assinatura de seus processos produtivos. A análise de conglomerados dos resultados de microanálise da fase wustita (usando como variáveis críticas os teores de $\mathrm{MnO}$, $\mathrm{MgO}, \mathrm{Al}_{2} \mathrm{O}_{3}, \mathrm{~V}_{2} \mathrm{O}_{5}$ and $\mathrm{TiO}_{2}$ ) mostrou-se efetiva para agrupar e diferenciar as amostras de diferentes origens: Bahia, Sardinha, Ipanema e Missões.

Palavras-chave: Análise de conglomerados; Arquemetalurgia; Inclusões nãometálicas; Artefatos ferroso; Assinatura de processo.

\section{MICROANALYSIS OF NON METALLIC INCLUSIONS IN IRON ARTIFACTS OF IPANEMA FACTORY AIMING TO ESTABLISH A CHEMICAL SIGNATURE OF ITS PRODUCTION PROCESS}

\section{Abstract}

Cluster analysis and microstructural characterization, including EDS microanalysis, were used to investigate the non-metallic inclusions ferrous samples of the Real Factory of Ipanema Iron and the D. Pedro II Bridge (located in Bahia and made in Scotland); and the ferrous artifacts from the archaeological sites of San Miguel of Missões (Rio Grande do Sul, Brazil) and Afonso Sardinha (São Paulo, Brazil, Prof. M. Andreatta's collection) in order to investigate the signature of their production processes. Cluster analysis of the results of the wustite phase of the inclusions (using as critical variables the contents $\mathrm{MnO}, \mathrm{MgO}, \mathrm{Al}_{2} \mathrm{O}_{3}, \mathrm{~V}_{2} \mathrm{O}_{5}$ and $\mathrm{TiO}_{2}$ ) was very effective in separating the four groups of samples: Bahia, Sardinha, Ipanema e Missões.

Keywords: Cluster analysis; Archaeometallurgy; Non-metallic inclusions; Ferrous artifacts; Provenance.

1 Professor Associado do Departamento de Engenharia de Materiais da Universidade de San Agustín, Arequipa, Perú; Mestrando em Engenharia Metalúrgica e de Materiais, Escola Politécnica Universidade de São Paulo, São Paulo, SP, Brasil.

2 Doutor em Engenharia Metalúrgica, Professor Associado do Departamento de Engenharia Metalúrgica e de Materiais, Escola Politécnica Universidade de São Paulo, São Paulo, SP, Brasil; e Presidente do Instituto de Pesquisas Tecnológicas do Estado de São Paulo S.A, São Paulo, SP, Brasil.

$3 \quad$ PhD, Professor Doutor do Departamento de Engenharia Metalúrgica e de Materiais, Escola Politécnica Universidade de São Paulo, São Paulo, SP, Brasil. 


\section{INTRODUÇÃO}

A Real Fábrica de Ferro de São João de Ipanema, localizada na cidade de Iperó no Estado de São Paulo, foi fundada em 1810 e seus altos fornos iniciaram a produção de ferro gusa em 1818. A fábrica produziu milhares de toneladas de barras de ferro, inicialmente pelo método de redução direta e depois exclusivamente por redução indireta, de modo intermitente até o ano de 1895 [1,2]. Usando o minério da mesma fonte (morro de Araçoiaba), Afonso Sardinha produziu algumas toneladas de ferro por redução direta no final do século XVI. Peças ferrosas produzidas antes do século XX contém grande quantidade de inclusões não-metálicas. A composição química dessas inclusões é influenciada pela composição das diferentes materiais, insumos e matérias-primas usadas durante o processo de manufatura (minério, cinzas de carvão, paredes dos fornos, fluxantes e etc.) [3]. Deste modo, a investigação da composição química das inclusões não-metálicas presentes em artefatos metálicos antigos pode servir para determinar algumas características tecnológicas e históricas sobre o processamento metalúrgico.

Dillmann e L'Héritier apresentaram em 2007 [3] uma metodologia com processamento de dados da microanálise EDS (energy dispersive spectrometry) de inclusões de 170 objetos ferrosos retirados de vários sítios arqueológicos, monumentos. A partir destes resultados, eles construíram gráficos bivariantes da porcentagem de alguns óxidos presentes nestas inclusões (por exemplo, $\% \mathrm{SiO}_{2}$ versus $\% \mathrm{Al}_{2} \mathrm{O}_{3}, \% \mathrm{CaO}$ versus $\% \mathrm{~K}_{2} \mathrm{O}, \% \mathrm{MgO}$ versus $\% \mathrm{Al}_{2} \mathrm{O}_{3}$ ) e, utilizando técnicas de filtragem de dados e de regressão linear passando pela origem dos respectivos gráficos, eles determinaram a "assinatura" do processo produtivo de cada um dos sítios analisados, identificada pelo coeficiente angular das correlações lineares. Adicionalmente, eles propuseram outro mapa bivariante de composição química das inclusões não metálicas $\left(\% \mathrm{P}_{2} \mathrm{O}_{5}\right.$ versus $\left.\left[\% \mathrm{Al}_{2} \mathrm{O}_{3}+\% \mathrm{MgO}+\% \mathrm{~K}_{2} \mathrm{O}\right] / \mathrm{FeO}\right)$ para identificar se os objetos ferrosos haviam sido produzidos por redução direta ou indireta.

Blakelock et al. [4] analisaram em 2009 vários corpos de prova (como lupas, pedaços de escória, barras e produtos forjados) de três experimentos de redução do minério de uma série produzida por T. Young (Museu of Welsh Life, Pais de Gales) entre 1998 e 2004. Nestes experimentos, foram recriadas as condições de produção artefatos de ferro produzidos pelos povos pré-históricos da região próxima de Cardiff, desde a redução direta de óxidos de ferro até o processo de forjamento (lupa -> barra $\rightarrow>$ forjamento) [5]. Adicionalmente, Blakelock et al. [4] compararam a composição química das inclusões não-metálicas de peças ferrosas provenientes de dois sítios arqueológicos distintos (Jordânia, século X d.C; e Israel, século IX a.C.) para tentar identificar a assinatura destes artefatos. Eles usaram várias combinações de gráficos bivariantes das composições químicas das inclusões e não conseguiram nem mesmo identificar a assinatura do processo produtivo dos artefatos ferrosos obtidos experimentalmente usando esta metodologia. Eles concluíram que a assinatura do processo dependia de inúmeras variáveis, como a composição química do minério, a técnica da fusão, a presença das cinzas do combustível, os parâmetros de funcionamento do forno, o uso e composição química dos fluxantes e dos materiais refratários dos fornos.

Desaulty et al. [6] examinaram em 2009 algumas hipóteses sobre as origens dos reforços de ferro de igrejas medievais francesas, usando análise química de elementos principais e de elementos traços de inclusões não-metálicas destes artefatos ferrosos. Eles usaram várias técnicas experimentais de análise química 
para analisar e comparar os resultados de 32 amostras procedentes das catedrais de Beauvais e Roven com os resultados de 14 amostras encontradas em sítios arqueológicos próximos da região de France Pays de Bray (região localizada entre as catedrais e famosa pela produção de ferro pelo processo de redução indireta durante o século $X V$ ). A análise dos resultados indicou que é possível usar elementos traços como identificadores de procedência de artefatos produzidos por redução direta. Finalmente os autores rejeitaram (por comparação) a hipótese de que as amostras de ferro retiradas das catedrais de Beauvais e Roven tinham sido produzidas na região de Pays de Bray.

Em 2012 Charlton et al. [7] analisaram os corpos de prova investigados por Blakelock et a. [4], usando uma nova metodologia que associava microanálise das inclusões com a análise de conglomerados para identificar padrões de comportamento de inclusões não-metálicas de componentes ferrosos. Adicionalmente, eles estudaram um caso prático com os dados compilados dos resultados de microanálise química das inclusões não metálicas de artefatos ferrosos provenientes da Escandinávia e obtidos por Buchwald [8] em 2005. Deste modo, Charlton et al. [7] identificaram a assinatura de objetos produzidos na Dinamarca, na Noruega e na Suécia.

Disser et al. [9] investigaram em 2014 as inclusões não-metálicas de 60 objetos ferrosos de reforços de monumentos góticos da Catedral de Beauvais (Picardie, França) e da Torre Mutte da Catedral de Metz (Lorraine, França). Eles aplicaram "análise de regressão logística" dos resultados de microanálise das inclusões para distinguir as peças ferrosas produzidas pelos processos de redução direta e redução indireta, e o método de análise multivariante para identificar a assinatura do processo produtivo dos artefatos ferrosos coletados nas catedrais góticas Francesas.

Em 2015 Maia et al. [10-12] analisaram por microanálise EDS as inclusões nãometálicas de objetos ferrosos coletados na Real Fábrica de Ferro de São João do Ipanema e no sítio de Sardinha. Foram construídos gráficos bivariantes da porcentagem de alguns óxidos presentes nas inclusões, como proposto por Dillmann e L'Héritier [3]. Eles mostraram as incongruências do método de regressão linear passando pela origem proposto por Dillmann e L'Héritier [3], mas não conseguiram identificar as assinaturas dos processos produtivos dos sítios examinados, apenas sugerindo que a presença de inclusões ricas em $\mathrm{TiO}_{2}$ seria uma característica típica dos artefatos coletados no sítio de Sardinha.

Objetiva-se no o presente trabalho desenvolver uma metodologia para identificar a assinatura química das peças de Ipanema, usando a análise microestrutural das inclusões de escória e a análise de aglomerados para separar os resultados em quatro grupos de resultados, compatíveis com suas procedências.

\section{MATERIAIS E MÉTODOS}

As amostras analisadas são parte da coleção de objetos que estão atualmente armazenados no Laboratório de Caracterização Microestrutural Hubertus Colpaert, da Escola Politécnica da Universidade de São Paulo, Brasil. Os objetos da série "Ipanema" são amostras da Coleção Geológica do Museu Nacional do Rio de Janeiro, que foram presentes do diretor da Fábrica de Ferro de Ipanema, o Coronel Mursa, para o Imperador Pedro II no ano de 1886 [13]. Os objetos de Ipanema devem ter sido produzidos pelo Refino Estiriano, descrito por Dupré [14]. A identificação e classificação destas amostras são mostradas na Tabela 1. 
Para cada amostra ferrosa, foi analisado por microanálise EDS um mínimo de 60 regiões (pontos $\mathrm{e}$ áreas) em inclusões não-metálicas e seus respectivos componentes microestruturais (fase matriz e wustita). A microanálise EDS foi realizada no microscópio eletrônico de varredura JEOL, JSM-6300 com detector da NORAN, usando uma distância de trabalho de $15 \mathrm{~mm}$, uma tensão aceleradora de $20 \mathrm{kV}$, modo área e tempo de aquisição de 180 segundos. A correção ZAF dos resultados de análise química (modo standardless) foi realizada e impondo como restrição para a análise química quantitativa, que todos os elementos químicos presentes nas inclusões estavam todos na forma de óxidos.

Tabela 1. Características e origem das amostras ferrosas analisadas

\begin{tabular}{|c|c|c|}
\hline Amostra & Origem & Descrição \\
\hline Ip-130 & Ipanema & Barra de seção retangular 10X4 mm \\
\hline Ip-131 & Ipanema & Barra de 8 mm de diâmetro \\
\hline Ip-133 & Ipanema & Barra de seção quadrada de 12X12 mm \\
\hline Ip-134 & Ipanema & Barra com seção quadrada de 17X17 mm \\
\hline Ip-135 & Ipanema & Barra seção quadrada de 22x22 mm \\
\hline B-145 & Escócia, Bahia & Seção de alma de ponte \\
\hline M-123 & $\begin{array}{c}\text { Missões } \\
\text { (Rio Grande do Sul ) }\end{array}$ & Prego de 3 cm de comprimento \\
\hline S-107 & Sardinha & Prego de 10 cm de comprimento \\
\hline
\end{tabular}

Para analisar o agrupamento das inclusões não metálicas e dos artefatos ferrosos de diversas origens foi utilizada a técnica de análise de conglomerados, que é uma técnica hierárquica que se baseia na ordenação e agrupamentos de resultados por distâncias euclidianas [15]. Os dados são agrupados sequencialmente, em cada etapa, formando-se um novo grupo que envolve dois ou mais grupos precedentes, segundo uma escala de distância entre grupos e os resultados são mostrados através de dendrogramas. Todos os dados foram organizados e manipulado no software MS Excel e analisado estatisticamente no software Statgraphics Centurion XVI version 16.1.03. (StatPoints Technologies Inc. 1982-2010).

\section{RESULTADOS}

Todas as amostras analisadas apresentaram teores de $\mathrm{FeO}$ acima de $60 \%$ na "inclusão inteira", como mostra a tabela 2. Os resultados indicam que o teor de $\mathrm{V}_{2} \mathrm{O}_{5}$ é maior para as amostras de Ipanema e de Sardinha. Adicionalmente, a amostra de Sardinha apresentou maiores teores de $\mathrm{MgO}$, enquanto Missões apresentou maior teor de $\mathrm{SiO}_{2}$. A análise microestrutural das inclusões não metálicas é mostrada nas Figuras 1-a, até 1-d. Em geral estas inclusões apresentam uma microestrutura do tipo dupléx com presença de dendritas de wustita $(\mathrm{FeO})$ em matriz "vítrea".

$\mathrm{Na}$ tabela 3 são mostradas as médias dos resultados de microanalise química para fase matriz das inclusões não-metálicas, sugerindo as amostras de Ipanema, com exceção da amostra I-135, apresentam, quando comparadas à amostra de Sardinha, $\mathrm{S}-107$, menores teores de $\mathrm{SiO}_{2}, \mathrm{~K}_{2} \mathrm{O}$ e $\mathrm{CaO}$ e maior teor de $\mathrm{P}_{2} \mathrm{O}_{5}$. Adicionalmente, a amostra Ip-135 apresentou resultados próximos aos da amostra de Sardinha. 
Tabela 2. Media dos microanálises por EDS do volume total da inclusão

\begin{tabular}{|c|c|c|c|c|c|c|c|c|c|c|}
\hline Sample & $\mathrm{MgO}$ & $\mathrm{SiO}_{2}$ & $\mathbf{P}_{2} \mathrm{O}_{5}$ & $\mathrm{~K}_{2} \mathrm{O}$ & $\mathrm{CaO}$ & $\mathrm{TiO}_{2}$ & $\mathrm{~V}_{2} \mathrm{O}_{5}$ & $\mathrm{MnO}$ & $\mathrm{Al}_{2} \mathrm{O}_{3}$ & $\mathrm{FeO}$ \\
\hline Ip-130 & $0.4 \pm 0.5$ & $6 \pm 6$ & $9 \pm 9$ & $0.4 \pm 0.4$ & $5 \pm 5$ & $0.4 \pm 0.3$ & $1.2 \pm 0.6$ & $0.6 \pm 0.4$ & $0.1 \pm 0.1$ & $78 \pm 17$ \\
\hline Ip-131 & $0.5 \pm 0.3$ & $10 \pm 5$ & $16 \pm 7$ & $0.5 \pm 0.5$ & $5 \pm 5$ & $0.5 \pm 0.5$ & $2 \pm 2$ & $0.8 \pm 0.4$ & $0.1 \pm 0.1$ & $64 \pm 12$ \\
\hline Ip-133 & $0.5 \pm 0.3$ & $8 \pm 6$ & $10 \pm 8$ & $0.4 \pm 0.3$ & $6 \pm 4$ & $0.5 \pm 0.5$ & $2 \pm 2$ & $0.6 \pm 0.2$ & $0.1 \pm 0.1$ & $73 \pm 16$ \\
\hline Ip-134 & $0.3 \pm 0.3$ & $5 \pm 4$ & $15 \pm 9$ & $0.2 \pm 0.2$ & $2 \pm 2$ & $0.3 \pm 0.3$ & $0.9 \pm 0.7$ & $0.5 \pm 0.3$ & $0.1 \pm 0.1$ & $77 \pm 9$ \\
\hline Ip-135 & $0.6 \pm 0.3$ & $12 \pm 12$ & $5 \pm 5$ & $0.6 \pm 0.5$ & $12 \pm 12$ & $0.6 \pm 0.3$ & $0.9 \pm 0.6$ & $0.5 \pm 0.3$ & $0.2 \pm 0.2$ & $71 \pm 27$ \\
\hline B-145 & $0.1 \pm 0.1$ & $10 \pm 4$ & $8 \pm 6$ & $0.1 \pm 0.1$ & $0.2 \pm 0.2$ & $0.6 \pm 0.3$ & $0.3 \pm 0.2$ & $2 \pm 1$ & $1.6 \pm 0.5$ & $77 \pm 9$ \\
\hline M-123 & $0.6 \pm 0.5$ & $19 \pm 16$ & $10 \pm 6$ & $1 \pm 1$ & $4 \pm 3$ & $0.1 \pm 0.3$ & $0.2 \pm 0.2$ & $0.6 \pm 0.4$ & $1.5 \pm 1.3$ & $63 \pm 18$ \\
\hline S-107 & $1.1 \pm 0.3$ & $15 \pm 6$ & $5 \pm 4$ & $2 \pm 1$ & $9 \pm 4$ & $0.9 \pm 0.5$ & $2 \pm 1$ & $1.3 \pm 0.3$ & $0.6 \pm 0.3$ & $64 \pm 12$ \\
\hline
\end{tabular}

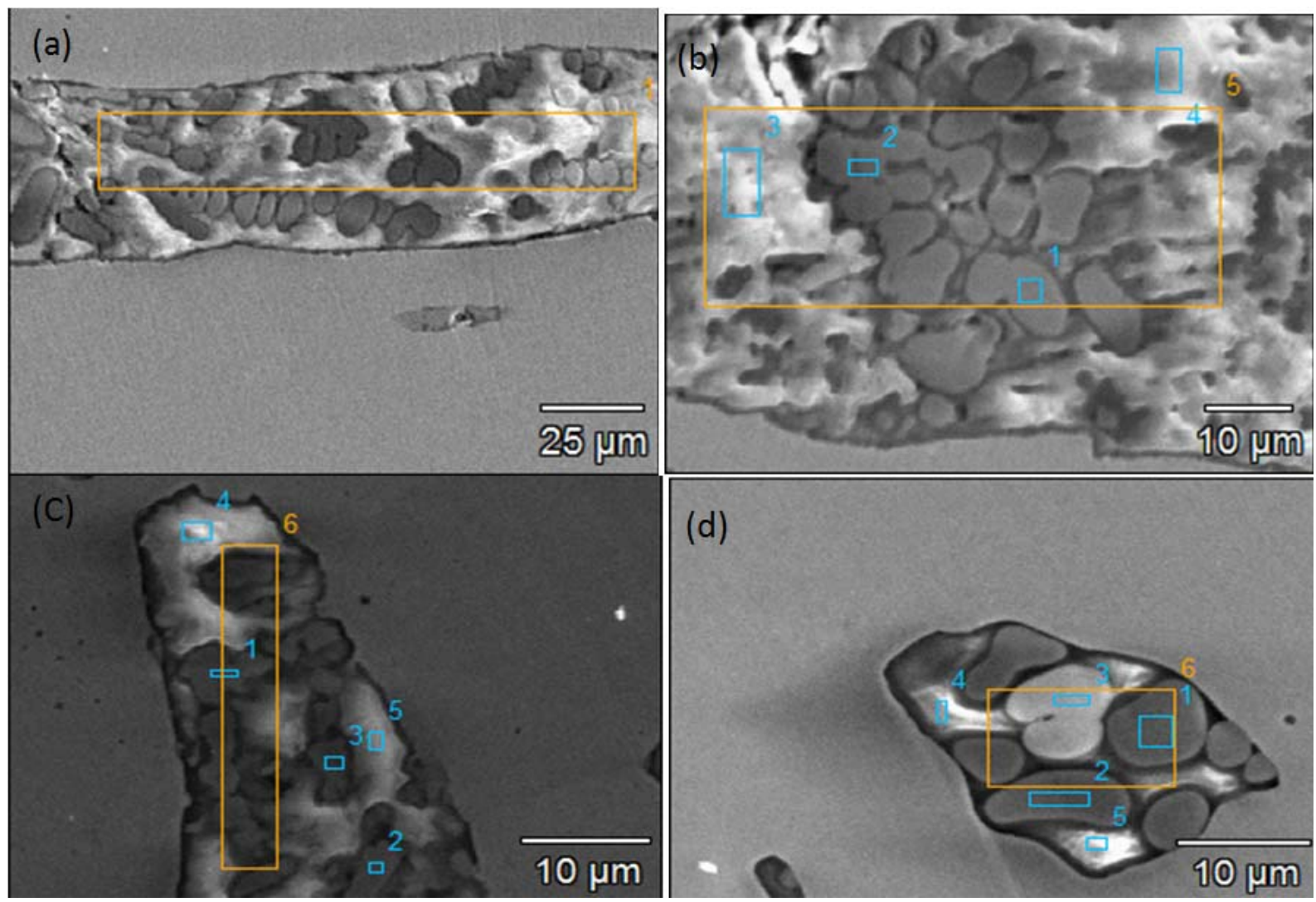

Figura 1. Microestrutura das inclusões não metálicas. (a) Ipanema (amostra Ip-135); (b) Bahia (amostra B-45); (c) Missões (amostra M-123); (d) Sardinha (amostra S107). 
Tabela 3. Médias dos resultados de microanalises EDS na matriz das inclusões.

\begin{tabular}{|c|c|c|c|c|c|c|c|c|c|c|}
\hline Sample & $\mathbf{M g O}$ & $\mathrm{SiO}_{2}$ & $\mathbf{P}_{2} \mathrm{O}_{5}$ & $\mathrm{~K}_{2} \mathrm{O}$ & $\mathrm{CaO}$ & $\mathrm{TiO}_{2}$ & $\mathbf{V}_{2} \mathrm{O}_{5}$ & $\mathrm{Al}_{2} \mathrm{O}_{3}$ & $\mathbf{M n O}$ & $\mathbf{F e O}$ \\
\hline Ip-130 & $0.6 \pm 0.2$ & $10 \pm 7$ & $22 \pm 9$ & $0.8 \pm 0.6$ & $9 \pm 5$ & $0.1 \pm 0.1$ & $0.1 \pm 0.1$ & $0.2 \pm 0.2$ & $0.7 \pm 0.2$ & $57 \pm 7$ \\
\hline Ip-131 & $1.0 \pm 0.4$ & $18 \pm 3$ & $17 \pm 4$ & $0.9 \pm 0.6$ & $9 \pm 6$ & $0.1 \pm 0.1$ & $0.1 \pm 0.1$ & $0.2 \pm 0.2$ & $1.0 \pm 0.1$ & $53 \pm 8$ \\
\hline Ip-133 & $0.8 \pm 0.2$ & $17 \pm 5$ & $19 \pm 5$ & $0.9 \pm 0.7$ & $10 \pm 6$ & $0.1 \pm 0.1$ & $0.1 \pm 0.1$ & $0.3 \pm 0.3$ & $0.8 \pm 0.2$ & $52 \pm 8$ \\
\hline Ip-134 & $0.9 \pm 0.3$ & $16 \pm 4$ & $17 \pm 4$ & $0.8 \pm 0.7$ & $7 \pm 4$ & $0.1 \pm 0.1$ & $0.1 \pm 0.1$ & $0.2 \pm 0.2$ & $0.9 \pm 0.2$ & $58 \pm 6$ \\
\hline Ip-135 & $0.8 \pm 0.3$ & $24 \pm 7$ & $7 \pm 5$ & $1.1 \pm 0.6$ & $14 \pm 8$ & $0.4 \pm 0.3$ & $0.2 \pm 0.2$ & $0.6 \pm 0.3$ & $0.8 \pm 0.4$ & $51 \pm 12$ \\
\hline B-145 & $0.1 \pm 0.1$ & $18 \pm 4$ & $13 \pm 6$ & $0.1 \pm 0.1$ & $0.4 \pm 0.3$ & $0.2 \pm 0.2$ & $0.1 \pm 0.1$ & $2 \pm 1$ & $3.4 \pm 0.7$ & $63 \pm 2$ \\
\hline M-123 & $0.4 \pm 0.0$ & $17 \pm 2$ & $13 \pm 3$ & $0.8 \pm 0.1$ & $4.2 \pm 0.3$ & $0.1 \pm 0.1$ & $0.1 \pm 0.1$ & $0.7 \pm 0.3$ & $0.5 \pm 0.1$ & $64 \pm 5$ \\
\hline S-107 & $1.4 \pm 0.3$ & $25 \pm 5$ & $9 \pm 4$ & $2.3 \pm 0.9$ & $15 \pm 5$ & $0.4 \pm 0.2$ & $0.2 \pm 0.2$ & $0.9 \pm 0.4$ & $1.5 \pm 0.3$ & $45 \pm 8$ \\
\hline
\end{tabular}

As microanálises químicas da fase wustita (região com alto teor de $\mathrm{FeO}$ ) de todas as amostras são mostradas na tabela 4, indicando que as amostras de Ipanema e de Sardinha apresentam maiores teores de $\mathrm{V}_{2} \mathrm{O}_{5}$, enquanto os elevados teores de $\mathrm{P}_{2} \mathrm{O}_{5}$ e $\mathrm{SiO}_{2}$ são aparentemente relevantes para a identificação da amostra de Missões.

Tabela 4. Médias dos resultados de microanálises EDS na fase wustita.

\begin{tabular}{|c|c|c|c|c|c|c|c|c|c|}
\hline Sample & $\mathrm{MgO}$ & $\mathrm{SiO}_{2}$ & $\mathrm{P}_{2} \mathrm{O}_{5}$ & $\mathrm{CaO}$ & $\mathrm{TiO}_{2}$ & $\mathrm{~V}_{2} \mathrm{O}_{5}$ & $\mathrm{Al}_{2} \mathrm{O}_{3}$ & $\mathrm{MnO}$ & $\mathrm{FeO}$ \\
\hline Ip-130 & $0.2 \pm 0.2$ & $0.4 \pm 0.2$ & $0.1 \pm 0.1$ & $0.1 \pm 0.1$ & $0.5 \pm 0.2$ & $1.7 \pm 0.6$ & $0.1 \pm 0.1$ & $0.3 \pm 0.2$ & $97 \pm 1$ \\
\hline Ip-131 & $0.1 \pm 0.1$ & $0.6 \pm 0.3$ & $0.2 \pm 0.2$ & $0.2 \pm 0.2$ & $1.0 \pm 0.3$ & $3.0 \pm 0.6$ & $0.1 \pm 0.1$ & $0.4 \pm 0.2$ & $95 \pm 1$ \\
\hline Ip-133 & $0.2 \pm 0.2$ & $0.3 \pm 0.1$ & $0.1 \pm 0.1$ & $0.1 \pm 0.1$ & $0.3 \pm 0.2$ & $1.5 \pm 0.6$ & $0.1 \pm 0.1$ & $0.1 \pm 0.2$ & $98 \pm 1$ \\
\hline Ip-134 & $0.1 \pm 0.1$ & $0.5 \pm 0.5$ & $0.8 \pm 0.8$ & $0.1 \pm 0.1$ & $0.5 \pm 0.4$ & $2.0 \pm 0.7$ & $0.1 \pm 0.1$ & $0.2 \pm 0.2$ & $96 \pm 2$ \\
\hline Ip-135 & $0.2 \pm 0.1$ & $0.3 \pm 0.1$ & $0.1 \pm 0.1$ & $0.1 \pm 0.1$ & $0.6 \pm 0.3$ & $1.6 \pm 0.9$ & $0.1 \pm 0.1$ & $0.4 \pm 0.1$ & $97 \pm 2$ \\
\hline $\mathrm{B}-145$ & $0.1 \pm 0.1$ & $0.8 \pm 0.3$ & $0.1 \pm 0.1$ & $0.1 \pm 0.1$ & $0.9 \pm 0.1$ & $0.5 \pm 0.2$ & $0.5 \pm 0.1$ & $0.9 \pm 0.4$ & $96 \pm 1$ \\
\hline $\mathrm{M}-123$ & $0.1 \pm 0.1$ & $3 \pm 2$ & $4 \pm 3$ & $0.3 \pm 0.3$ & $0.1 \pm 0.1$ & $0.3 \pm 0.2$ & $0.6 \pm 0.3$ & $0.1 \pm 0.1$ & $92 \pm 5$ \\
\hline S-107 & $0.4 \pm 0.2$ & $0.9 \pm 0.4$ & $0.1 \pm 0.1$ & $0.3 \pm 0.2$ & $1.2 \pm 0.3$ & $3.2 \pm 0.9$ & $0.1 \pm 0.1$ & $0.7 \pm 0.1$ & $93 \pm 1$ \\
\hline
\end{tabular}

Um gráfico bivariante $\% \mathrm{TiO}_{2}$ versus $\% \mathrm{~V}_{2} \mathrm{O}_{5}$ com todos os resultados de microanálise da fase wustita (sugerido como resultado que diferencia as amostras de Sardinha e de Ipanema das demais) é mostrado na figura 2, indicando um agrupamento diferenciado dos resultados das amostras da Bahia e Missões. Não foi possível, no entanto, separar as amostras de Ipanema e Sardinha (que apresentam uma região de intersecção, e não podem ser diferenciadas espacialmente), embora exista uma tendência de as inclusões de sardinha apresentarem maiores teores de $\mathrm{TiO}_{2}$ e $\mathrm{V}_{2} \mathrm{O} 5$ do que as inclusões de Ipanema. 


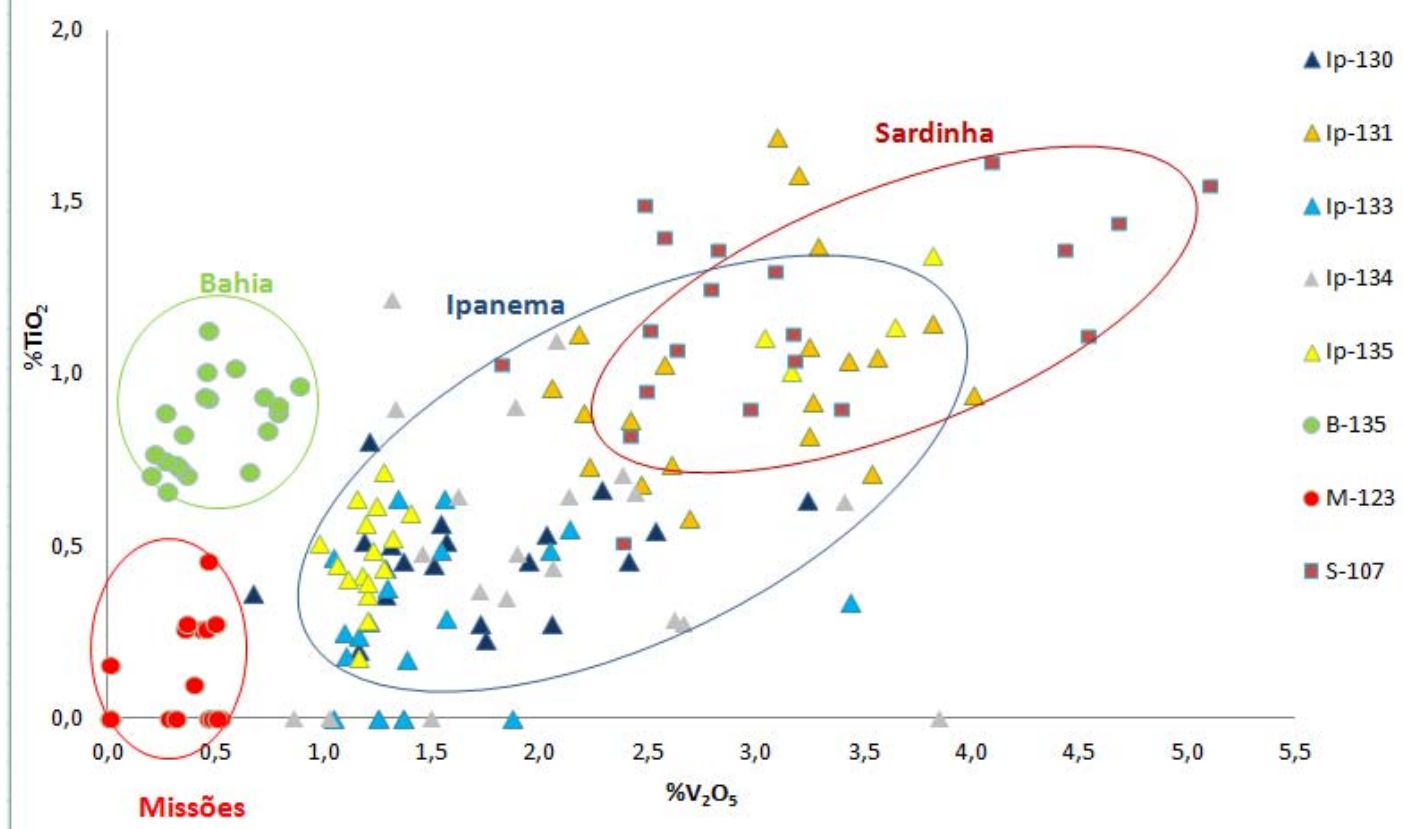

Figura 2. Gráfico bivariante $\% \mathrm{TiO}_{2}$ versus $\% \mathrm{~V}_{2} \mathrm{O}_{5}$, das amostras Ipanema, Bahia, Missões e Sardinha na fase wustita.

Para fazer um estudo mais detalhado da relação entre os resultados de microanálise química da fase wustita das inclusões não-metálicas e os seus respectivos sítios de coleta, utilizou-se a análise de conglomerados tendo como variáveis críticas os teores de $\mathrm{MnO}, \mathrm{MgO}, \mathrm{Al}_{2} \mathrm{O}_{3}, \mathrm{~V}_{2} \mathrm{O}_{5}$ and $\mathrm{TiO}_{2}$ na fase wustita (vide Figura 3). Foi traçada uma linha horizontal no dendrograma da Figura 3, sendo possível separar quatro conglomerados distintos de amostras, compatíveis com suas origens: Missões, Bahia, Ipanema e Sardinha. 


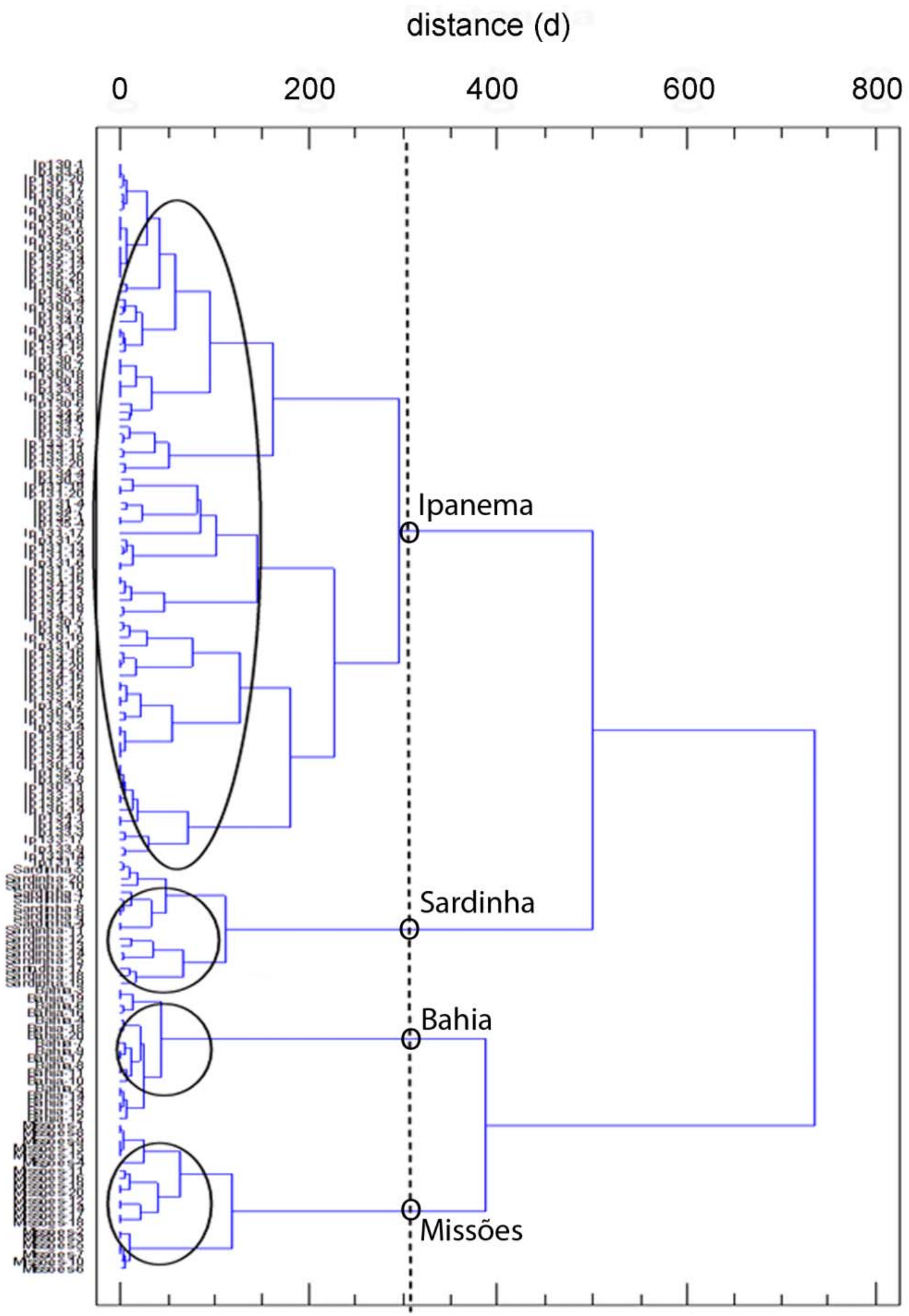

Figura 3. Dendograma do agrupamento das inclusões não metálicas de Ipanema, Sardinha, Missões e Bahia, utilizando como variáveis críticas os teores $\mathrm{MnO}, \mathrm{MgO}$, $\mathrm{Al}_{2} \mathrm{O}_{3}, \mathrm{~V}_{2} \mathrm{O}_{5}$ and $\mathrm{TiO}_{2}$ presentes na fase wustita. 


\section{DISCUSSÃO}

Os resultados de microanálise da fase wustita relevaram a presença de maiores teores de $\mathrm{V}_{2} \mathrm{O}_{5}$ para as amostras de Ipanema e de Sardinha e de maiores teores de $\mathrm{P}_{2} \mathrm{O}_{5}$ e $\mathrm{SiO}_{2}$ para a amostra de Missões. $\mathrm{O}$ gráfico bivariante de $\mathrm{TiO}_{2}$ versus $\mathrm{V}_{2} \mathrm{O}_{5}$ (vide Figura 2) permitiu o agrupamento dos conjuntos de objetos ferrosos e a identificação de três grupos distintos, não sendo possível separar as inclusões de Sardinha das inclusões das amostras de Ipanema, embora exista uma tendência de as inclusões de sardinha apresentarem maiores teores de $\mathrm{TiO}_{2}$ e $\mathrm{V}_{2} \mathrm{O} 5$ do que as inclusões de Ipanema.

Foi proposta uma nova metodologia para separar e agrupar as amostras ferrosas de diferentes proveniências. Esta metodologia associou os resultados de microanálise da fase wustita das inclusões não-metálicas com a análise de conglomerados usando como variáveis críticas os teores de $\mathrm{MnO}, \mathrm{MgO}, \mathrm{Al}_{2} \mathrm{O}_{3}, \mathrm{~V}_{2} \mathrm{O}_{5}$ e $\mathrm{TiO}_{2}$ para a construção de um dendrograma (vide Figura 3 ), que permitiu separar com sucesso os quatro grupos distintos de grupos de resultados: Bahia, Sardinha, Ipanema e Missões.

\section{CONCLUSÕES}

1. Os resultados de microanálise química por EDS das amostras de Ipanema e Sardinha indicam que o vanádio fica concentrado nas dendritas da fase wustita das inclusões.

2. Os altos teores de $\mathrm{TiO}_{2}$ e $\mathrm{V}_{2} \mathrm{O}_{5}$ nas dendritas da fase wustita das inclusões de não metálicas são características típicas da microestrutura dos artefatos ferrosos da Fábrica de Ferro de Ipanema e do Sítio Sardinha.

3. A análise de conglomerados nos resultados de microanálise da fase wustita (usando como variáveis críticas os teores de $\mathrm{MnO}, \mathrm{MgO}_{2} \mathrm{Al}_{2} \mathrm{O}_{3}, \mathrm{~V}_{2} \mathrm{O}_{5}$ e $\mathrm{TiO}_{2}$ ) mostrou-se efetiva para agrupar e diferenciar as amostras de diferentes origens: Bahia, Sardinha, Ipanema e Missões.

\section{Agradecimentos}

Os autores F. J. G. Landgraf e C. R. F. Azevedo agradecem bolsa de pesquisa do CNPq. Os autores agradecem ao Centro de Tecnologia em Metalurgia e Materiais do Instituto de Pesquisas Tecnológicas (IPT) e ao Laboratório de Microscopia do Departamento de Engenharia Metalúrgica e de Materiais da Escola Politécnica da Universidade de São Paulo pelo apoio aos trabalhos de microscopia eletrônica e microanálise EDS..

\section{REFERÊNCIAS}

1. Baer, W. The development of the Brazilian steel industry. Vanderbilt University Press, 202 p., 1969.

2. Santos N. P. A Fabrica de ferro São João de Ipanema: economia e política nas últimas décadas do Segundo Reinado (1860-1889). Dissertação de Mestrado, Faculdade de Filosofia, Letras e Ciência Humanas, Universidade de São Paulo, 180 p., 2009.

3. Dillmann, P.; M. L'Héritier. Slag inclusion analyses for studying ferrous alloys employed in French medieval buildings: supply of materials and diffusion of smelting processes. Journal of Archaeological Science. Vol. 34, p. 1810-1823, 2007. 
4. Blakelock, E.; Martinon-Torres M.; Veldhuijzen V.H.; Young T. Slag inclusions in iron objects and the quest for provenance: an experiment and a case study. Journal of Archaeological Science. Vol.36, p. 1745-1757, 2009.

5. Crew, P. The experimental production of Prehistoric bar iron. Historical Metallurgy. Vol. 25, 21-36, 1991

6. Desaulty, A-M., Dillmann, P.; L'Heritiera, M.; Mariet, C.; Gratuze, B.; Joron, J-L.; Fluzin, P. Does it come from the Pays de Bray? Examination of an origin hypothesis for the ferrous reinforcements used in French medieval churches using major and trace element analyses. Journal of Archaeological Science. Vol. 36, p. 2445-2462, 2009.

7. Charlton F.; Blakelock, E.; Martinón-Torres M.; Young, T. Investigating the production provenance of iron artifacts with multivariate methods. Journal of Archaeological Science. Vol. 39, p. 2280-2293, 2012.

8. Buchwald, V.F. Iron and Steel in Ancient Times. Editado por: The Royal Danish Academy of Sciences and Letters, Copenhagen. 372 p., 2005.

9. Disser, A.; Dillmann, P.; Bourgain, C.; L'Héritier, M.; Vega, E.; Bauvais, S., Leroy, M. Iron reinforcements in Beauvais and Metz Cathedrals: from bloomery or finery? The use of logistic regression for differentiating smelting processes. Journal of Archaeological Science. Vol. 42, p. 315-333, 2014.

10. Maia, R.R.; Dias, M.S.; Azevedo, C.R.F.; Landgraf, F.J.G. Archaeometry of ferrous artefacts from Luso-Brazilian archaeological sites near Ipanema River, Brazil. REM, Revista Escola de Minas, Vol.2, 187-193, 2015.

11. Maia R.R. Análise de inclusões de escória em amostras Arqueológicas da fábrica de ferro de Ipanema, Dissertação de Mestrado. Escola Politécnica, Universidade de São Paulo, 209 p., 2014.

12. Maia, R.R.; Azevedo, C.R.F.; Landgraf, F.J.G. Análise de inclusões de escória em amostras da fábrica de ferro de Ipanema. $67^{\circ}$ Congresso ABM Internacional, 2012, Rio de Janeiro.

13. Landgraf, F.J.G., Tschiptschin, A. P.; Goldenstein, H. Notas sobre a história da metalurgia no Brasil (1500-1850), ed. UNESP, São Paulo, 1995.

14. Dupré, L. Memória sobre a Fábrica de Ferro de São João do Ipanema. Anaes da Escola de Minas de Ouro Preto. N. 4. Typografia D'O Germinal. Mariana: 1885. p.49.

15. Johnson R., Wichern D. Applied Multivariate Statistical Analysis. Sixth edition. Pearson Education Inc., 2007. 